Supplement of Biogeosciences, 13, 4751-4765, 2016

http://www.biogeosciences.net/13/4751/2016/

doi:10.5194/bg-13-4751-2016-supplement

(C) Author(s) 2016. CC Attribution 3.0 License.

(c) (i)

Supplement of

\title{
Effects of wastewater treatment plant effluent inputs on planktonic metabolic rates and microbial community composition in the Baltic Sea
}

Raquel Vaquer-Sunyer et al.

Correspondence to: Raquel Vaquer-Sunyer (raquel.vaquer@uib.cat)

The copyright of individual parts of the supplement might differ from the CC-BY 3.0 licence. 
Table S1. Nutrients (in $\mu \mathrm{M})$ and metabolic rates $\left(\mathrm{mmol} \mathrm{O}_{2} \mathrm{~m}^{-3} \mathrm{~d}^{-1}\right)$ for all days and treatments for the four experiments.

\begin{tabular}{|c|c|c|c|c|c|c|c|c|c|c|c|c|c|c|c|c|c|c|c|c|c|c|c|c|c|c|c|c|c|c|}
\hline Experiment & Day & Sample & $\begin{array}{l}\text { Mean } \\
\text { TDN } \\
(\mu \mathrm{M}) \\
\end{array}$ & SE & $\begin{array}{l}\text { Mean } \\
\mathrm{NO}_{2}^{-} \\
\end{array}$ & $\mathrm{SE}$ & $\begin{array}{l}\text { Mean } \\
\mathrm{NO}_{3}^{-} \\
\end{array}$ & SE & $\mathrm{NH} 4$ & SE & DON & SE & $\begin{array}{l}\text { Mean } \\
\text { DPA } \\
(\mu \mathrm{M}) \\
\end{array}$ & SE & $\begin{array}{l}\text { Mean } \\
\mathrm{PO}_{4}{ }^{3-} \\
\end{array}$ & SE & $\mathrm{NCP}$ & SD & GPP & SD & $\mathrm{CR}$ & SD & Temp. & SD & $\begin{array}{c}\text { Mean BP } \\
(\mu \mathrm{g} \mathrm{C} \mathrm{L-1} \\
\mathrm{h}-1)\end{array}$ & SE & $\begin{array}{l}\text { Mean } \\
\text { Chl.a } \\
(\mu \mathrm{g} / 1) \\
\end{array}$ & $\mathrm{SE}$ & $\begin{array}{l}\text { DOC } \\
(\mu \mathrm{M}) \\
\end{array}$ & SE \\
\hline WWTP-S & T0 & $\mathrm{C}$ & 16.51 & 0.08 & 0.01 & 0.00 & 0.50 & 0.09 & 0.24 & 0.00 & 15.76 & 0.12 & 0.17 & 0.01 & 0.03 & 0.01 & 15.40 & 9.26 & 42.82 & 11.20 & -27.41 & 1.94 & & & 5.98 & 0.34 & 6.49 & 0.01 & & \\
\hline WWTP-S & T0 & $\mathrm{CD}$ & 12.59 & 0.29 & & & 0.35 & 0.02 & 0.49 & 0.07 & 11.74 & 0.30 & 0.21 & 0.05 & 0.02 & 0.00 & 16.92 & 4.64 & 43.01 & 7.87 & -26.09 & 3.23 & & & 6.02 & 0.69 & 7.12 & 0.11 & 274.30 & 2.30 \\
\hline WWTP-S & T0 & $1: 10$ & 64.04 & 0.88 & 2.92 & 0.05 & 28.87 & 0.45 & 13.26 & 0.15 & 19.00 & 0.99 & 0.50 & 0.07 & 0.05 & 0.00 & 23.12 & 4.24 & 47.36 & 2.89 & -24.24 & 7.12 & & & 5.15 & 0.46 & 6.98 & 0.19 & 387.42 & 2.03 \\
\hline WWTP-S & T0 & $1: 5$ & 110.89 & 2.11 & 4.79 & 0.13 & 46.84 & 1.32 & 26.82 & 1.13 & 32.44 & 2.73 & 0.63 & 0.03 & 0.10 & 0.01 & 22.78 & 5.78 & 46.74 & 2.29 & -23.96 & 8.07 & & & 4.60 & 0.53 & 5.21 & 0.10 & & \\
\hline WWTP-S & T0 & IN & 48.84 & 0.97 & 6.48 & 0.03 & 27.33 & 0.68 & 0.55 & 0.09 & 14.49 & 1.19 & 0.10 & 0.04 & 0.11 & 0.00 & 11.73 & 3.01 & 33.41 & 2.93 & -21.68 & 5.94 & & & 4.16 & 0.35 & 6.50 & 0.31 & 272.07 & 3.18 \\
\hline WWTP-S & $\mathrm{T} 1$ & $\mathrm{C}$ & 17.63 & 0.46 & 0.03 & 0.02 & 0.32 & 0.06 & 0.16 & 0.02 & 17.12 & 0.46 & 0.15 & 0.01 & 0.03 & 0.01 & 3.22 & 1.13 & 35.81 & 2.26 & -32.59 & 1.12 & 18.49 & 0.00 & 3.77 & 0.20 & 6.49 & 0.08 & 340.19 & 1.87 \\
\hline WWTP-S & $\mathrm{T} 1$ & $\mathrm{CD}$ & 12.01 & 1.05 & 0.03 & 0.00 & 0.44 & 0.05 & 0.17 & 0.02 & 11.37 & 1.05 & 0.12 & 0.00 & 0.02 & 0.00 & 5.56 & 1.68 & 31.10 & 10.25 & -25.55 & 8.57 & 18.49 & 0.00 & 4.14 & 0.35 & 6.52 & 0.38 & 270.34 & 3.50 \\
\hline WWTP-S & $\mathrm{T} 1$ & $1: 10$ & 63.72 & 0.41 & 3.00 & 0.02 & 29.66 & 0.55 & 12.30 & 0.16 & 18.76 & 0.70 & 0.44 & 0.04 & 0.04 & 0.01 & 1.78 & 2.87 & 29.76 & 12.84 & -27.98 & 9.97 & 18.49 & 0.00 & 5.97 & 0.73 & 4.83 & 0.01 & 390.36 & 1.41 \\
\hline WWTP-S & $\mathrm{T} 1$ & $1: 5$ & 113.07 & 1.30 & 5.90 & 0.01 & 58.96 & 2.77 & 21.01 & 3.58 & 27.19 & 4.71 & 0.59 & 0.01 & 0.05 & 0.01 & 3.83 & 1.62 & 33.80 & 2.17 & -29.97 & 3.78 & 18.49 & 0.00 & 7.86 & 0.85 & 5.31 & 0.02 & 494.36 & 1.76 \\
\hline WWTP-S & $\mathrm{T} 1$ & IN & 45.50 & 0.59 & 6.07 & 0.20 & 25.98 & 1.13 & 0.12 & 0.02 & 13.33 & 1.26 & 0.12 & 0.01 & 0.06 & 0.02 & 3.95 & 0.44 & 26.87 & 7.20 & -22.92 & 6.76 & 18.49 & 0.00 & 3.51 & 1.92 & 5.92 & 0.22 & 271.36 & 2.69 \\
\hline WWTP-S & $\mathrm{T} 3$ & $\mathrm{C}$ & 17.93 & 0.39 & & & 0.35 & 0.02 & 0.42 & 0.04 & 17.17 & 0.39 & 0.14 & 0.01 & 0.04 & 0.01 & 2.28 & 0.82 & 37.17 & 2.18 & -34.89 & 1.35 & 18.50 & 0.00 & 4.28 & 0.22 & 5.63 & 0.05 & & \\
\hline WWTP-S & $\mathrm{T} 3$ & $\mathrm{CD}$ & 12.92 & 0.21 & & & 0.51 & 0.11 & 0.15 & 0.05 & 12.26 & 0.24 & 0.19 & 0.08 & 0.03 & 0.01 & 1.47 & 1.69 & 31.85 & 2.84 & -30.38 & 1.15 & 18.50 & 0.00 & 3.63 & 0.43 & 4.89 & 0.16 & 271.87 & 5.45 \\
\hline WWTP-S & $\mathrm{T} 3$ & $1: 10$ & 62.08 & 0.48 & 2.91 & 0.01 & 28.60 & 0.35 & 10.46 & 0.13 & 20.11 & 0.61 & 0.28 & 0.04 & 0.03 & 0.01 & 9.51 & 0.91 & 47.45 & 6.57 & -37.94 & 5.66 & 18.50 & 0.00 & 4.24 & 0.20 & 8.83 & 0.15 & 387.63 & 1.76 \\
\hline WWTP-S & T3 & $1: 5$ & 107.64 & 0.76 & 5.86 & 0.02 & 59.08 & 1.03 & 24.24 & 0.22 & 18.45 & 1.30 & 0.56 & 0.03 & 0.03 & 0.01 & 9.86 & 0.04 & 43.71 & 9.20 & -33.85 & 9.17 & 18.50 & 0.00 & 6.62 & 0.87 & 9.37 & 0.07 & 494.77 & 1.43 \\
\hline WWTP-S & $\mathrm{T} 3$ & IN & 44.58 & 1.36 & 6.22 & 0.04 & 25.40 & 1.01 & 0.09 & 0.03 & 12.87 & 1.70 & 0.10 & 0.01 & 0.02 & 0.00 & 10.39 & 1.03 & 45.27 & 10.96 & -34.88 & 9.93 & 18.50 & 0.00 & 3.35 & 0.15 & 9.43 & 0.07 & 268.82 & 1.02 \\
\hline WWTP-S & T5 & $\mathrm{C}$ & 17.09 & 0.29 & 0.02 & 0.01 & 0.32 & 0.03 & 0.60 & 0.01 & 16.16 & 0.29 & 0.18 & 0.01 & 0.03 & 0.01 & 3.83 & 3.62 & 36.19 & 4.64 & -32.36 & 1.02 & 18.37 & 0.00 & 5.25 & 0.79 & 5.90 & 0.00 & 349.01 & 2.42 \\
\hline WWTP-S & $\mathrm{T} 5$ & $\mathrm{CD}$ & 13.74 & 0.39 & 0.01 & 0.00 & 0.42 & 0.02 & 0.03 & 0.01 & 13.28 & 0.39 & 0.15 & 0.02 & 0.02 & 0.00 & 4.74 & 0.24 & 35.68 & 4.05 & -30.93 & 3.81 & 18.37 & 0.00 & 2.83 & 0.21 & 5.31 & 0.11 & 275.01 & 1.79 \\
\hline WWTP-S & T5 & $1: 10$ & 59.98 & 0.54 & 2.73 & 0.02 & 28.52 & 1.03 & 9.32 & 0.12 & 19.41 & 1.17 & 0.31 & 0.01 & 0.03 & 0.00 & 10.89 & 0.57 & 42.75 & 4.15 & -31.86 & 4.72 & 18.37 & 0.00 & 5.83 & 0.16 & 9.38 & 0.36 & 484.84 & 1.51 \\
\hline WWTP-S & T5 & $1: 5$ & 105.59 & 1.78 & 5.61 & 0.00 & 58.79 & 1.40 & -0.47 & 0.06 & 41.66 & 2.26 & 0.49 & 0.03 & 0.03 & 0.00 & 15.23 & 2.63 & 56.52 & 1.11 & -41.29 & 1.52 & 18.37 & 0.00 & 8.06 & 0.76 & 11.27 & 0.08 & 628.47 & 1.20 \\
\hline WWTP-S & T5 & IN & 41.75 & 0.18 & 5.83 & 0.02 & 22.92 & 0.34 & 18.70 & 0.56 & -5.70 & 0.68 & 0.09 & 0.02 & 0.03 & 0.01 & 15.35 & 1.69 & 52.71 & 6.12 & -37.36 & 4.43 & 18.37 & 0.00 & 5.71 & 0.16 & 11.48 & 0.57 & 488.08 & 2.38 \\
\hline WWTP-S & $\mathrm{T} 7$ & $\mathrm{C}$ & 16.77 & 0.63 & 0.01 & 0.01 & 0.36 & 0.05 & 0.01 & 0.00 & 16.40 & 0.63 & 0.12 & 0.03 & 0.03 & 0.00 & 20.17 & 5.78 & 54.16 & 5.31 & -33.99 & 11.09 & 18.33 & 0.04 & 2.84 & 0.20 & 6.41 & 0.30 & 483.62 & 3.64 \\
\hline WWTP-S & $\mathrm{T} 7$ & $\mathrm{CD}$ & 13.99 & 1.03 & & & 0.33 & 0.03 & 0.06 & 0.03 & 13.60 & 1.03 & 0.11 & 0.01 & 0.03 & 0.00 & 8.20 & 7.29 & 36.11 & 11.34 & -27.91 & 4.05 & 18.33 & 0.04 & 2.10 & 0.16 & 5.12 & 0.37 & 345.86 & 0.90 \\
\hline WWTP-S & $\mathrm{T} 7$ & $1: 10$ & 58.39 & 0.42 & 2.50 & 0.06 & 28.36 & 0.96 & 8.27 & 0.11 & 19.26 & 1.06 & 0.31 & 0.01 & 0.04 & 0.01 & 15.59 & 19.91 & 46.15 & 23.02 & -30.56 & 3.11 & 18.33 & 0.04 & 3.95 & 0.77 & 9.68 & 0.14 & 554.98 & 12.74 \\
\hline
\end{tabular}




\begin{tabular}{|c|c|c|c|c|c|c|c|c|c|c|c|c|c|c|c|c|c|c|c|c|c|c|c|c|c|c|c|c|c|c|}
\hline Experiment & Day $\mathrm{s}$ & Sample & $\begin{array}{l}\text { Mean } \\
\text { TDN } \\
(\mu \mathrm{M})\end{array}$ & $\mathrm{SE}$ & $\begin{array}{l}\text { Mean } \\
\mathrm{NO}_{2}^{-}\end{array}$ & $\mathrm{SE}$ & $\begin{array}{l}\text { Mean } \\
\mathrm{NO}_{3}^{-}\end{array}$ & SE & $\mathrm{NH}_{4}^{+}$ & SE & DON & SE & $\begin{array}{l}\text { Mean } \\
\text { DPA } \\
(\mu \mathrm{M})\end{array}$ & SE & $\begin{array}{l}\text { Mean } \\
\mathrm{PO}_{4}{ }^{3-}\end{array}$ & $\mathrm{SE}$ & $\mathrm{NCP}$ & $\mathrm{SD}$ & GPP & SD & $\mathrm{CR}$ & SD & Temp. & SD & $\begin{array}{c}\text { Mean BP } \\
\text { ( } \mu \text { g C L-1 } \\
\text { h-1) }\end{array}$ & $\mathrm{SE}$ & $\begin{array}{l}\text { Mean } \\
\text { Chl.a } \\
(\mu \mathrm{g} / \mathrm{l})\end{array}$ & $\mathrm{SE}$ & $\begin{array}{l}\text { DOC } \\
(\mu \mathrm{M})\end{array}$ & SE \\
\hline WWTP-S & $\mathrm{T} 7$ & $1: 5$ & 101.79 & 1.71 & 5.31 & 0.01 & 57.37 & 0.52 & 19.07 & 0.25 & 20.04 & 1.81 & 0.49 & 0.01 & 0.03 & 0.01 & 27.31 & 9.77 & 77.98 & 14.45 & -50.67 & 4.68 & 18.33 & 0.04 & 5.85 & 0.51 & 15.23 & 0.34 & & \\
\hline WWTP-S & $\mathrm{T} 7$ & IN & 39.64 & 0.42 & 5.69 & 0.01 & 21.18 & 0.22 & 0.01 & & 12.76 & 0.48 & 0.10 & 0.02 & 0.04 & 0.00 & 36.69 & 1.49 & 85.67 & 7.13 & -48.97 & 5.64 & 18.34 & 0.04 & 4.52 & 0.91 & 10.45 & 0.25 & 327.21 & 27.23 \\
\hline WW-F & $\mathrm{T} 0$ & $\mathrm{C}$ & 20.99 & 0.34 & 0.21 & 0.05 & 2.64 & 0.32 & 0.23 & 0.03 & 17.91 & 0.47 & 0.24 & 0.03 & 0.39 & 0.02 & & & & & & & & & 0.39 & 0.04 & 1.76 & 0.04 & 318.45 & 9.42 \\
\hline WW-F & T0 & $\mathrm{CD}$ & 16.84 & 0.37 & 0.21 & 0.02 & 2.57 & 0.26 & 0.47 & 0.01 & 13.59 & 0.45 & 0.19 & 0.02 & 0.50 & 0.03 & & & & & & & & & 0.29 & 0.03 & 1.66 & 0.12 & 245.16 & 3.20 \\
\hline WW-F & T0 & $1: 10$ & 66.17 & 0.69 & 2.34 & 0.06 & 31.44 & 0.73 & 16.84 & 0.19 & 15.55 & 1.02 & 0.56 & 0.10 & 0.65 & 0.02 & & & & & & & & & 0.37 & 0.05 & 1.64 & 0.27 & 320.71 & 1.28 \\
\hline WW-F & T0 & $1: 5$ & 114.85 & 1.26 & 2.97 & 0.10 & 42.80 & 1.77 & 31.29 & 0.23 & 37.79 & 2.18 & 0.78 & 0.04 & 0.47 & 0.02 & & & & & & & & & 0.37 & 0.01 & 1.60 & 0.04 & 393.78 & 3.56 \\
\hline WW-F & T0 & IN & 63.11 & 0.44 & 2.89 & 0.15 & 30.75 & 1.80 & 0.21 & 0.01 & 29.26 & 1.85 & 0.03 & 0.01 & 0.40 & 0.03 & & & & & & & & & 0.32 & 0.02 & 1.30 & 0.11 & 274.08 & 14.45 \\
\hline WW-F & $\mathrm{T} 1$ & $\mathrm{C}$ & 19.98 & 0.37 & 0.30 & 0.01 & 3.43 & 0.22 & 0.08 & 0.01 & 16.17 & 0.43 & & & 0.70 & 0.08 & -8.83 & & 14.61 & & -23.44 & & 7.69 & 0.13 & 0.59 & 0.04 & 1.16 & 0.08 & 299.29 & 2.72 \\
\hline WW-F & $\mathrm{T} 1$ & $\mathrm{CD}$ & 16.85 & 0.27 & 0.22 & 0.04 & 2.66 & 0.29 & 0.23 & 0.01 & 13.74 & 0.39 & & & 0.46 & 0.09 & -10.86 & 0.08 & 13.31 & 1.96 & -24.17 & 1.88 & 7.78 & 0.00 & 0.57 & 0.03 & 0.90 & 0.06 & 285.17 & 22.24 \\
\hline WW-F & $\mathrm{T} 1$ & $1: 10$ & 58.25 & 3.82 & 2.09 & 0.05 & 29.62 & 1.26 & 16.43 & 0.13 & 10.11 & 4.03 & & & 0.56 & 0.03 & -10.30 & & 9.03 & & -19.04 & 0.41 & 7.69 & 0.13 & 0.69 & 0.02 & 0.89 & 0.14 & 434.00 & 10.94 \\
\hline WW-F & $\mathrm{T} 1$ & $1: 5$ & 117.31 & 1.88 & 3.45 & 0.23 & 51.58 & 3.51 & 28.97 & 0.86 & 33.31 & 4.06 & & & 0.51 & 0.03 & -9.49 & & 6.59 & & -17.24 & 1.63 & 7.69 & 0.14 & 0.61 & 0.03 & 0.82 & 0.03 & 429.91 & 5.41 \\
\hline WW-F & $\mathrm{T} 1$ & IN & 63.81 & 0.56 & 4.26 & 0.06 & 49.16 & 0.87 & 0.18 & 0.02 & 10.21 & 1.03 & & & 0.60 & 0.03 & -10.15 & 2.49 & 5.28 & 1.41 & -15.43 & 1.08 & 7.78 & 0.00 & 0.56 & 0.06 & 0.70 & 0.07 & 291.84 & 25.46 \\
\hline WW-F & $\mathrm{T} 3$ & $\mathrm{C}$ & 19.99 & 0.59 & 0.28 & 0.05 & 3.20 & 0.26 & 0.05 & 0.01 & 16.46 & 0.64 & 0.14 & 0.04 & 0.68 & 0.07 & 2.89 & 1.64 & 22.38 & 3.02 & -19.48 & 1.39 & 7.18 & 0.00 & 0.66 & 0.05 & 1.48 & 0.05 & 297.31 & 6.59 \\
\hline WW-F & T3 & $\mathrm{CD}$ & 15.47 & 0.76 & 0.12 & 0.02 & 2.24 & 0.10 & 0.09 & 0.01 & 13.02 & 0.76 & 0.16 & 0.01 & 0.32 & 0.05 & 1.76 & 1.21 & 21.09 & 0.85 & -19.32 & 0.36 & 7.18 & 0.00 & 1.47 & 0.21 & 1.19 & 0.01 & 292.95 & 6.68 \\
\hline WW-F & $\mathrm{T} 3$ & 1:10 & 68.07 & 0.72 & 1.89 & 0.38 & 27.53 & 6.32 & 16.17 & 0.14 & 22.49 & 6.35 & 0.34 & 0.01 & 0.49 & 0.11 & 1.44 & 0.77 & 18.12 & 0.09 & -16.68 & 0.68 & 7.18 & 0.00 & 1.86 & 0.49 & 1.52 & 0.04 & 311.10 & 6.53 \\
\hline WW-F & $\mathrm{T} 3$ & $1: 5$ & 116.68 & 0.84 & 3.12 & 0.02 & 47.73 & 1.13 & 31.29 & 0.33 & 34.53 & 1.45 & 0.70 & 0.03 & 0.45 & 0.02 & 1.25 & 0.46 & 14.79 & 1.05 & -13.54 & 0.59 & 7.18 & 0.00 & 2.54 & 0.25 & 1.54 & 0.10 & 361.20 & 4.06 \\
\hline WW-F & $\mathrm{T} 3$ & IN & 61.72 & 1.20 & 2.53 & 0.48 & 28.50 & 5.42 & -0.02 & 0.01 & 30.72 & 5.53 & 0.03 & 0.01 & 0.32 & 0.08 & 0.36 & 0.37 & 10.56 & 1.81 & -10.20 & 1.44 & 7.18 & 0.00 & 1.14 & 0.04 & 0.97 & 0.04 & 227.31 & 2.33 \\
\hline WW-F & T5 & $\mathrm{C}$ & 18.74 & 0.53 & 0.30 & 0.01 & 3.44 & 0.06 & 0.29 & 0.01 & 14.72 & 0.53 & 0.15 & 0.03 & 0.86 & 0.02 & 2.23 & 1.17 & 25.77 & 0.54 & -23.54 & 0.64 & 7.27 & 0.00 & 0.54 & 0.03 & 2.19 & 0.06 & 284.40 & 1.80 \\
\hline WW-F & T5 & $\mathrm{CD}$ & 15.63 & 0.22 & 0.23 & 0.03 & 2.59 & 0.18 & 0.26 & 0.00 & 12.55 & 0.28 & 0.10 & 0.01 & 0.54 & 0.05 & 2.90 & 0.08 & 27.08 & 1.63 & -24.17 & 1.71 & 7.27 & 0.00 & 1.03 & 0.04 & 1.61 & 0.11 & 248.63 & 10.06 \\
\hline WW-F & T5 & $1: 10$ & 66.36 & 0.46 & 2.57 & 0.02 & 38.24 & 0.78 & 12.27 & 2.53 & 13.29 & 2.69 & 0.36 & 0.03 & 0.60 & 0.01 & 2.68 & 1.11 & 24.23 & 3.39 & -21.55 & 2.28 & 7.27 & 0.00 & 1.84 & 0.09 & 3.65 & 0.20 & 294.46 & 2.23 \\
\hline WW-F & T5 & $1: 5$ & 117.00 & 0.86 & 5.05 & 0.06 & 80.66 & 2.08 & 30.36 & 0.50 & 0.93 & 2.31 & 0.65 & 0.01 & 0.69 & 0.04 & 3.24 & 0.47 & 20.29 & 0.10 & -17.06 & 0.57 & 7.27 & 0.00 & 3.08 & 0.37 & 3.47 & 0.14 & 379.90 & 3.11 \\
\hline WW-F & T5 & IN & 63.35 & 0.45 & 3.83 & 0.67 & 44.56 & 8.75 & 0.04 & 0.02 & 14.92 & 8.74 & 0.05 & 0.02 & 0.52 & 0.10 & 2.24 & 2.27 & 15.68 & 1.79 & -13.44 & 0.49 & 7.27 & 0.00 & 0.96 & 0.03 & 1.29 & 0.11 & 228.92 & 1.62 \\
\hline
\end{tabular}




\begin{tabular}{|c|c|c|c|c|c|c|c|c|c|c|c|c|c|c|c|c|c|c|c|c|c|c|c|c|c|c|c|c|c|c|}
\hline xperiment & Day & Sample & $\begin{array}{l}\text { Mean } \\
\text { TDN } \\
(\mu \mathrm{M})\end{array}$ & $\mathrm{SE}$ & $\begin{array}{l}\text { Mean } \\
\mathrm{NO}_{2}^{-}\end{array}$ & $\mathrm{SE}$ & $\begin{array}{l}\text { Mean } \\
\mathrm{NO}_{3}^{-}\end{array}$ & SE & $\mathrm{NH}_{4}^{+}$ & SE & DON & SE & $\begin{array}{c}\text { Mean } \\
\text { DPA } \\
(\mu \mathrm{M})\end{array}$ & SE & $\begin{array}{l}\text { Mean } \\
\mathrm{PO}_{4}{ }^{3-}\end{array}$ & SE & $\mathrm{NCP}$ & SD & GPP & SD & $\mathrm{CR}$ & SD & Temp. & SD & $\begin{array}{c}\text { Mean BP } \\
(\mu \mathrm{g} \mathrm{C} \mathrm{L}-1 \\
\mathrm{h}-1)\end{array}$ & SE & $\begin{array}{l}\text { Mean } \\
\text { Chl.a } \\
(\mu \mathrm{g} / \mathrm{l})\end{array}$ & SE & $\begin{array}{l}\text { DOC } \\
(\mu \mathrm{M})\end{array}$ & SE \\
\hline WW-F & $\mathrm{T} 7$ & $\mathrm{C}$ & 17.21 & 0.61 & 0.14 & 0.00 & 1.97 & 0.34 & 0.06 & 0.00 & 15.04 & 0.70 & 0.12 & 0.08 & 0.69 & 0.00 & 17.83 & 10.29 & 43.69 & 3.98 & -25.86 & 6.30 & 7.16 & 0.07 & 0.54 & 0.04 & 4.87 & 0.21 & 307.88 & 15.57 \\
\hline WW-F & $\mathrm{T} 7$ & $\mathrm{CD}$ & 14.42 & 0.16 & 0.10 & 0.01 & 1.89 & 0.04 & 0.16 & 0.01 & 12.28 & 0.16 & 0.22 & 0.08 & 0.56 & 0.02 & 14.46 & 4.54 & 43.99 & 4.76 & -29.53 & 9.30 & 7.16 & 0.07 & 0.82 & 0.09 & 4.15 & 0.11 & 230.57 & 2.75 \\
\hline WW-F & $\mathrm{T} 7$ & $1: 10$ & 61.17 & 1.23 & 2.43 & 0.07 & 35.16 & 1.09 & 11.39 & 3.17 & 12.19 & 3.57 & 0.35 & 0.01 & 0.43 & 0.02 & 24.34 & 15.71 & 60.27 & 9.93 & -35.94 & 25.64 & 7.16 & 0.07 & 1.15 & 0.07 & 8.53 & 0.38 & 308.80 & 5.90 \\
\hline WW-F & $\mathrm{T} 7$ & $1: 5$ & 109.23 & 4.55 & 4.64 & 0.04 & 73.25 & 3.25 & 27.90 & 0.13 & 3.44 & 5.59 & 0.43 & 0.07 & 0.49 & 0.03 & 19.46 & 9.87 & 73.62 & 45.72 & -54.16 & 55.59 & 7.16 & 0.07 & 1.66 & 0.10 & 9.25 & 0.35 & 367.72 & 3.67 \\
\hline WW-F & $\mathrm{T} 7$ & IN & 63.34 & 0.98 & 4.66 & 0.06 & 54.92 & 0.37 & & & 3.76 & 1.04 & 0.09 & 0.00 & 0.58 & 0.02 & 9.86 & 7.88 & 41.84 & 20.24 & -31.99 & 28.12 & 7.16 & 0.07 & 0.88 & 0.07 & 3.08 & 0.34 & 232.41 & 5.44 \\
\hline WW-J & T0 & $\mathrm{C}$ & 17.07 & 0.87 & 0.35 & 0.02 & 4.93 & 0.39 & 0.35 & 0.01 & 11.44 & 0.95 & 0.09 & 0.01 & 0.55 & 0.03 & & & & & & & & & 0.15 & 0.05 & 0.30 & 0.00 & 483.13 & 68.40 \\
\hline WW-J & T0 & $\mathrm{CD}$ & 13.10 & 0.80 & 0.31 & 0.01 & 3.36 & 0.06 & 0.27 & 0.02 & 9.15 & 0.80 & 0.08 & 0.01 & 0.48 & 0.01 & & & & & & & & & 0.09 & 0.06 & 0.59 & 0.05 & 339.56 & 123.9 \\
\hline WW-J & T0 & $1: 10$ & 101.14 & 1.95 & 1.08 & 0.06 & 11.68 & 0.88 & 75.23 & 0.59 & 13.15 & 2.22 & 0.97 & 0.03 & 0.77 & 0.07 & & & & & & & & & 0.06 & 0.01 & 0.30 & 0.00 & 467.47 & 20.41 \\
\hline WW-J & T0 & $1: 5$ & 174.65 & 3.08 & 2.16 & 0.18 & 27.23 & 0.35 & 129.69 & 3.16 & 15.56 & 4.42 & 1.78 & 0.03 & 1.30 & 0.09 & & & & & & & & & 0.05 & 0.01 & 0.34 & 0.01 & 570.45 & 35.83 \\
\hline WW-J & T0 & IN & 35.21 & 1.31 & 3.17 & 0.21 & 24.91 & 2.74 & 2.22 & 0.07 & 4.92 & 3.03 & 0.11 & 0.01 & 0.72 & 0.10 & & & & & & & & & 0.04 & 0.01 & 0.36 & 0.02 & 357.57 & 82.45 \\
\hline WW-J & $\mathrm{T} 1$ & $\mathrm{C}$ & 18.65 & 0.67 & 0.38 & 0.02 & 4.65 & 0.69 & 0.45 & 0.01 & 13.17 & 0.96 & 0.09 & 0.01 & 0.66 & 0.08 & -2.84 & 5.76 & 4.17 & 2.23 & -7.01 & 3.53 & 3.52 & 0.00 & 0.13 & 0.02 & 0.15 & 0.04 & 490.20 & 7.58 \\
\hline WW-J & $\mathrm{T} 1$ & $\mathrm{CD}$ & 13.18 & 1.18 & 0.31 & 0.03 & 3.17 & 0.08 & 0.37 & 0.06 & 9.34 & 1.18 & 0.10 & 0.01 & 0.46 & 0.02 & -8.64 & 10.10 & -3.72 & 8.81 & -4.92 & 1.28 & 3.53 & 0.00 & 0.09 & 0.01 & 0.22 & 0.08 & 385.03 & 6.44 \\
\hline WW-J & $\mathrm{T} 1$ & $1: 10$ & 94.87 & 2.24 & 0.93 & 0.21 & 8.32 & 3.18 & 74.94 & 1.13 & 10.68 & 4.04 & 0.89 & 0.03 & 0.61 & 0.22 & -16.64 & 17.69 & -12.51 & 16.31 & -4.13 & 1.38 & 3.49 & 0.06 & 0.11 & 0.04 & 0.24 & 0.04 & 522.23 & 5.19 \\
\hline WW-J & $\mathrm{T} 1$ & $1: 5$ & 191.30 & 1.79 & 2.07 & 0.20 & 20.58 & 1.56 & 137.57 & 1.63 & 31.08 & 2.88 & 1.81 & 0.05 & 1.50 & 0.05 & -2.76 & 1.75 & 0.20 & 2.20 & -2.96 & 0.45 & 3.53 & 0.00 & 0.08 & 0.03 & 0.24 & 0.05 & 647.05 & 8.99 \\
\hline WW-J & $\mathrm{T} 1$ & IN & 31.22 & 2.01 & 2.64 & 0.14 & 24.23 & 0.27 & 2.06 & 0.02 & 2.29 & 2.02 & 0.11 & 0.01 & 0.41 & 0.04 & -0.79 & 3.14 & 0.15 & 1.77 & -0.95 & 1.37 & 3.53 & 0.00 & 0.10 & 0.01 & 0.20 & 0.01 & 383.44 & 5.72 \\
\hline WW-J & $\mathrm{T} 3$ & $\mathrm{C}$ & 20.16 & 0.37 & 0.38 & 0.02 & 4.81 & 0.34 & 0.17 & 0.01 & 14.81 & 0.50 & 0.03 & 0.01 & 0.61 & 0.04 & -4.00 & 1.97 & 5.88 & 0.93 & -9.88 & 2.89 & 3.58 & 0.00 & 0.31 & 0.14 & 0.16 & 0.00 & 369.97 & 89.08 \\
\hline WW-J & $\mathrm{T} 3$ & $\mathrm{CD}$ & 15.46 & 0.21 & 0.35 & 0.02 & 3.30 & 0.10 & 0.26 & 0.03 & 11.55 & 0.23 & 0.05 & 0.01 & 0.41 & 0.03 & 1.09 & 0.06 & 6.79 & 0.35 & -5.71 & 0.41 & 3.58 & 0.00 & 0.23 & 0.03 & 0.28 & 0.04 & 347.99 & 87.75 \\
\hline WW-J & $\mathrm{T} 3$ & $1: 10$ & 103.04 & 1.92 & 1.37 & 0.02 & 11.63 & 0.70 & 71.02 & 1.24 & 19.03 & 2.39 & 0.94 & 0.02 & 0.89 & 0.14 & 0.36 & 1.53 & 6.51 & 1.47 & -6.15 & 0.05 & 3.58 & 0.00 & 0.23 & 0.03 & 0.29 & 0.02 & 453.15 & 59.10 \\
\hline WW-J & $\mathrm{T} 3$ & $1: 5$ & 184.83 & 3.92 & 2.00 & 0.18 & 27.87 & 0.66 & 134.52 & 2.45 & 20.45 & 4.66 & 0.09 & 0.00 & 1.14 & 0.08 & -0.42 & 0.65 & 6.53 & 0.89 & -6.95 & 1.54 & 3.58 & 0.00 & 0.24 & 0.02 & 0.22 & & 570.89 & 93.60 \\
\hline WW-J & $\mathrm{T} 3$ & IN & 35.77 & 0.29 & 2.85 & 0.02 & 30.41 & 1.78 & 2.01 & 0.01 & 0.50 & 1.81 & 1.86 & 0.04 & 0.43 & 0.01 & -1.22 & 0.34 & 5.01 & 1.78 & -6.23 & 1.43 & 3.58 & 0.00 & 0.15 & 0.01 & 0.07 & & 314.44 & 5.26 \\
\hline WW-J & T5 & $\mathrm{C}$ & 15.39 & 1.58 & 0.39 & 0.02 & 4.52 & 0.19 & 0.18 & 0.01 & 10.29 & 1.59 & 0.07 & 0.01 & 0.69 & 0.10 & -3.27 & 1.01 & 2.03 & 2.00 & -5.30 & 0.99 & 3.64 & 0.01 & 0.55 & 0.20 & 0.35 & 0.01 & 418.52 & 34.02 \\
\hline WW-J & T5 & $\mathrm{CD}$ & 14.94 & 0.24 & 0.37 & 0.03 & 3.45 & 0.40 & 0.02 & 0.00 & 11.10 & 0.47 & 0.06 & 0.01 & 0.44 & 0.09 & -2.94 & 0.04 & 1.22 & 0.53 & -4.17 & 0.56 & 3.65 & 0.00 & 0.40 & 0.02 & 0.28 & 0.08 & 312.04 & 19.34 \\
\hline
\end{tabular}




\begin{tabular}{|c|c|c|c|c|c|c|c|c|c|c|c|c|c|c|c|c|c|c|c|c|c|c|c|c|c|c|c|c|c|c|}
\hline xperiment & Day & Sample & $\begin{array}{l}\text { Mean } \\
\text { TDN } \\
(\mu \mathrm{M})\end{array}$ & $\mathrm{SE}$ & $\begin{array}{l}\text { Mean } \\
\mathrm{NO}_{2}^{-}\end{array}$ & $\mathrm{SE}$ & $\begin{array}{l}\text { Mean } \\
\mathrm{NO}_{3}^{-}\end{array}$ & SE & $\mathrm{NH}_{4}^{+}$ & SE & DON & SE & $\begin{array}{l}\text { Mean } \\
\text { DPA } \\
(\mu \mathrm{M})\end{array}$ & SE & & SE & NCP & SD & GPP & SD & CR & SD & Temp. & SD & $\begin{array}{c}\text { Mean BP } \\
(\mu \mathrm{g} \mathrm{C} \mathrm{L-1} \\
\mathrm{h}-1)\end{array}$ & SE & $\begin{array}{l}\text { Mean } \\
\text { Chl.a } \\
(\mu \mathrm{g} / 1)\end{array}$ & SE & $\begin{array}{l}\text { DOC } \\
(\mu \mathrm{M})\end{array}$ & SE \\
\hline WW-J & T5 & $1: 10$ & 94.20 & 1.16 & 1.26 & 0.02 & 13.57 & 0.70 & 68.23 & 4.30 & 11.15 & 4.51 & 0.97 & 0.03 & 0.87 & 0.04 & -4.76 & 0.16 & 0.14 & 1.91 & -4.90 & 1.75 & 3.65 & 0.00 & 0.55 & 0.04 & 0.35 & 0.04 & 497.34 & 104.0 \\
\hline WW-J & T5 & $1: 5$ & 188.49 & 3.16 & 1.78 & 0.12 & 26.49 & 0.82 & 121.77 & 3.39 & 38.46 & 4.71 & 1.70 & 0.05 & 1.12 & 0.05 & -3.31 & 1.40 & -0.37 & 0.24 & -2.94 & 1.64 & 3.65 & 0.00 & 0.65 & 0.04 & 0.29 & 0.01 & 906.24 & 93.29 \\
\hline WW-J & T5 & IN & 35.67 & 0.33 & 3.33 & 0.23 & 33.05 & 1.40 & 1.84 & 0.03 & -2.54 & 1.42 & 0.05 & 0.02 & 0.71 & 0.20 & -3.00 & 1.65 & 8.19 & 10.66 & -11.19 & 9.01 & 3.65 & 0.00 & 0.29 & 0.04 & 0.21 & 0.05 & 321.31 & 33.29 \\
\hline WW-J & $\mathrm{T} 7$ & $\mathrm{C}$ & 17.55 & 0.78 & 0.41 & 0.01 & 3.74 & 0.15 & 0.40 & 0.42 & 13.01 & 0.90 & 0.07 & 0.00 & 0.50 & 0.06 & 4.18 & 1.05 & 19.16 & 8.81 & -14.98 & 9.87 & 3.72 & 0.01 & 062 & 0.02 & 0.36 & 0.01 & 446.54 & 57.58 \\
\hline WW-J & $\mathrm{T} 7$ & $\mathrm{CD}$ & 15.55 & 0.16 & 0.37 & 0.04 & 4.16 & 0.23 & 0.09 & 0.01 & 10.92 & 0.27 & 0.04 & 0.00 & 0.42 & 0.02 & 10.07 & 9.13 & -34.15 & 40.27 & 44.22 & 49.40 & 3.72 & 0.00 & 1.38 & 0.69 & 0.26 & 0.03 & 418.79 & 201.9 \\
\hline WW-J & $\mathrm{T} 7$ & $1: 10$ & 99.55 & 2.89 & 1.40 & 0.05 & 17.44 & 0.95 & 76.98 & 1.14 & 3.72 & 3.25 & 0.96 & 0.21 & 0.89 & 0.06 & 2.74 & 4.03 & 11.03 & 17.02 & -8.28 & 21.05 & 3.72 & 0.00 & 112 & 0.03 & 0.32 & 0.04 & 699.62 & 13.68 \\
\hline WW-J & $\mathrm{T} 7$ & $1: 5$ & 194.64 & 1.29 & 2.16 & 0.18 & 24.89 & 0.86 & 134.58 & 5.56 & 33.01 & 5.77 & 1.94 & 0.05 & 1.08 & 0.11 & 0.76 & 1.07 & 4.68 & 5.96 & -3.92 & 4.89 & 3.72 & 0.00 & 1.14 & 0.07 & 0.26 & 0.02 & 844.06 & 10.78 \\
\hline WW-J & $\mathrm{T} 7$ & IN & 34.29 & 1.30 & 2.99 & 0.32 & 27.89 & 4.00 & 1.81 & 0.01 & 1.59 & 4.20 & 0.07 & 0.01 & 0.36 & 0.06 & 2.05 & 0.62 & 18.73 & 17.31 & -16.68 & 17.92 & 3.72 & 0.00 & 063 & 0.02 & 0.27 & 0.02 & 427.80 & 115.8 \\
\hline WW-A & T0 & $\mathrm{C}$ & 16.40 & 0.63 & .14 & 0.00 & 3.69 & 0.14 & 0.01 & 0.01 & 12.56 & 0.64 & 0.31 & 0.01 & 0.63 & 0.03 & & & & & & & & & 0.24 & 0.05 & 2.34 & 0.27 & 297.38 & 3.08 \\
\hline WW-A & T0 & $\mathrm{CD}$ & 12.72 & 0.47 & 0.12 & 0.01 & 3.29 & 0.06 & 0.14 & 0.01 & 9.17 & 0.48 & 0.22 & 0.03 & 0.51 & 0.03 & & & & & & & & & 0.17 & 0.01 & 1.86 & & 659.33 & 23.57 \\
\hline WW-A & T0 & $1: 10$ & 103.16 & 1.16 & .84 & 0.00 & 17.43 & 0.41 & 76.91 & 0.50 & $\begin{array}{c}7.97 \\
-\end{array}$ & 1.33 & 1.95 & 0.39 & 0.48 & 0.03 & & & & & & & & & & 0.01 & 2.35 & & & 47.67 \\
\hline WW-A & T0 & $1: 5$ & 160.50 & 4.19 & 1.52 & 0.00 & 30.06 & 2.08 & 146.16 & 1.24 & 17.25 & 7.47 & 3.69 & 0.06 & 0.55 & 0.02 & & & & & & & & & & 0.04 & 4.73 & 0.60 & 953.14 & 5.84 \\
\hline WW-A & $\mathrm{T} 1$ & $\mathrm{C}$ & 15.24 & 0.89 & 0.19 & 0.01 & 3.75 & 0.08 & 0.01 & 0.01 & 11.29 & & & & 0.90 & 0.07 & 0.78 & & & & & & & & & 0.01 & 4.52 & 0.13 & & \\
\hline WW-A & $\mathrm{T} 1$ & $\mathrm{CD}$ & 12.57 & 0.25 & 0.19 & 0.00 & 3.26 & 0.07 & 0.12 & 0.01 & 9.00 & 0.26 & & & 0.45 & 0.04 & 2.21 & & 7.51 & & -5.30 & & 4.55 & & 0.27 & 0.06 & 2.33 & 0.27 & & \\
\hline WW-A & $\mathrm{T} 1$ & $1: 10$ & 98.85 & 1.98 & 0.95 & 0.00 & 18.34 & 0.24 & 76.20 & 0.95 & 3.35 & 2.21 & & & 0.44 & 0.01 & 0.98 & 3.16 & 7.93 & 2.46 & -6.95 & 5.62 & & 0.00 & & 0.01 & 2.28 & 0.17 & & \\
\hline WW-A & $\mathrm{T} 1$ & $1: 5$ & 187.92 & 5.28 & 1.64 & 0.01 & 32.11 & 0.13 & 146.30 & 4.56 & 7.87 & 6.98 & & & 0.45 & 0.01 & & & & & & & & & 0.25 & 0.03 & 2.01 & 0.16 & & \\
\hline WW-A & $\mathrm{T} 1$ & IN & 55.76 & 0.88 & 1.77 & 0.00 & 37.02 & 2.85 & 4.48 & 0.21 & 12.48 & 2.99 & 0.23 & 0.01 & 1.35 & 0.04 & 1.22 & 0.33 & 22.61 & 3.79 & -21.39 & 4.12 & 4.55 & 0.00 & & 0.04 & 4.06 & 0.41 & 559.29 & 8.31 \\
\hline WW-A & $\mathrm{T} 3$ & $\mathrm{C}$ & 15.95 & 0.39 & 0.19 & 0.01 & 3.34 & 0.17 & & 0.03 & 12.31 & 0.43 & 0.14 & 0.01 & 0.53 & 0.02 & -3.99 & 6.42 & 20.28 & 8.09 & -24.27 & 1.67 & 4.55 & 0.00 & 0.63 & 0.02 & 5.93 & 0.27 & 759.37 & 5.92 \\
\hline WW-A & $\mathrm{T} 3$ & $\mathrm{CD}$ & 12.42 & 0.38 & 0.12 & 0.00 & 2.83 & 0.04 & 0.02 & 0.00 & 9.44 & 0.38 & 0.11 & 0.01 & 0.42 & 0.02 & -0.13 & & 22.42 & & -22.55 & & 4.55 & & 0.26 & 0.03 & 3.56 & 0.36 & 534.23 & 1.53 \\
\hline WW-A & $\mathrm{T} 3$ & $1: 10$ & 98.52 & 4.45 & 0.91 & 0.00 & 16.88 & 0.79 & 76.33 & 1.38 & 4.40 & 4.72 & 1.94 & 0.04 & 0.40 & 0.01 & 0.20 & 0.23 & 28.07 & 5.50 & -27.87 & 5.73 & 4.55 & 0.00 & 0.76 & 0.02 & 4.21 & 0.20 & 655.29 & 9.47 \\
\hline WW-A & $\mathrm{T} 3$ & $1: 5$ & 177.63 & 8.99 & 1.57 & 0.01 & 32.33 & 0.44 & 148.83 & 1.08 & -5.10 & 9.06 & & & 0.40 & 0.04 & 0.41 & & 22.43 & & -22.02 & & 4.55 & & 0.79 & 0.04 & 2.88 & 0.07 & 715.55 & 6.04 \\
\hline WW-A & T3 & IN & 52.52 & 0.12 & 1.63 & 0.09 & 40.46 & 0.52 & 3.92 & 0.07 & 6.51 & 0.53 & & & 1.32 & 0.03 & -2.75 & 0.05 & 10.46 & 0.21 & -13.21 & 0.16 & 4.46 & 0.00 & 0.77 & 0.06 & 6.43 & 0.78 & 606.74 & 2.58 \\
\hline
\end{tabular}




\begin{tabular}{|c|c|c|c|c|c|c|c|c|c|c|c|c|c|c|c|c|c|c|c|c|c|c|c|c|c|c|c|c|c|c|}
\hline Experiment & Day & Sample & $\begin{array}{l}\text { Mean } \\
\text { TDN } \\
(\mu \mathrm{M})\end{array}$ & $\mathrm{SE}$ & $\begin{array}{l}\text { Mean } \\
\mathrm{NO}_{2}^{-}\end{array}$ & SE & $\begin{array}{l}\text { Mean } \\
\mathrm{NO}_{3}\end{array}$ & $\mathrm{SE}$ & $\mathrm{NH}_{4}^{+}$ & SE & DON & SE & $\begin{array}{l}\text { Mean } \\
\text { DPA } \\
(\mu \mathrm{M})\end{array}$ & $\mathrm{SE}$ & $\begin{array}{l}\text { Mean } \\
\mathrm{PO}_{4}{ }^{3-}\end{array}$ & $\mathrm{SE}$ & $\mathrm{NCP}$ & SD & GPP & SD & $\mathrm{CR}$ & SD & Temp. & SD & $\begin{array}{c}\text { Mean BP } \\
(\mu \mathrm{g} \mathrm{C} \mathrm{L-1} \\
\mathrm{h}-1)\end{array}$ & SE & $\begin{array}{l}\text { Mean } \\
\text { Chl.a } \\
(\mu \mathrm{g} / \mathrm{l})\end{array}$ & SE & $\begin{array}{l}\text { DOC } \\
(\mu \mathrm{M})\end{array}$ & SE \\
\hline WW-A & T5 & $\mathrm{C}$ & 12.44 & 1.27 & 0.12 & 0.00 & 2.64 & 0.03 & 1.27 & 0.61 & 8.41 & 1.41 & 0.24 & 0.02 & 0.46 & 0.02 & -1.41 & 2.12 & 12.26 & 1.73 & -13.67 & 0.40 & 4.46 & 0.00 & 0.55 & 0.07 & 6.64 & 0.88 & 735.79 & 36.12 \\
\hline WW-A & T5 & CD & 13.14 & 1.13 & 0.09 & 0.00 & 2.73 & 0.04 & 0.05 & 0.01 & 10.26 & 1.13 & 0.25 & 0.01 & 0.44 & 0.04 & -2.89 & & 6.51 & & -9.40 & & 4.46 & & 0.97 & 0.01 & 4.14 & 1.09 & 835.92 & 58.43 \\
\hline WW-A & T5 & $1: 10$ & 102.73 & 3.19 & 0.85 & 0.00 & 17.83 & 0.24 & 74.61 & 2.16 & 9.43 & 3.86 & 1.78 & 0.04 & 0.30 & 0.01 & -2.07 & 1.82 & 4.33 & 0.18 & -6.40 & 1.64 & 4.46 & 0.00 & 1.07 & 0.08 & 6.54 & 0.69 & 980.53 & 29.25 \\
\hline WW-A & $\mathrm{T} 5$ & $1: 5$ & 189.21 & 3.81 & 1.53 & 0.03 & 24.25 & 9.71 & 147.34 & 0.91 & 16.09 & 10.47 & 3.67 & 0.06 & 0.33 & 0.02 & -3.17 & & 5.92 & & -9.09 & & 4.46 & & 1.21 & 0.04 & 4.99 & 0.09 & 1130.24 & 80.36 \\
\hline WW-A & T5 & IN & 52.47 & 1.26 & 1.70 & 0.00 & 39.76 & 0.86 & 3.10 & 0.02 & 7.91 & 1.53 & 0.27 & 0.01 & 1.21 & 0.01 & 14.50 & & 31.74 & & -17.24 & & 4.44 & & 1.08 & 0.10 & 7.96 & 0.88 & 917.62 & 29.18 \\
\hline WW-A & $\mathrm{T} 7$ & $\mathrm{C}$ & 15.73 & 1.09 & 0.12 & 0.00 & 2.62 & 0.14 & 0.54 & 0.20 & 12.44 & 1.12 & 0.26 & 0.01 & 0.48 & 0.02 & 11.00 & & 25.23 & & -14.23 & & 4.44 & & 0.88 & 0.06 & 6.49 & 0.39 & 293.67 & 1.10 \\
\hline WW-A & $\mathrm{T} 7$ & C & 14.13 & 0.28 & 0.11 & 0.01 & 2.38 & 0.03 & & & 11.64 & 0.28 & 0.20 & 0.03 & & & 4.93 & & 21.40 & & -16.47 & & 4.44 & & 0.46 & 0.01 & 7.54 & 0.46 & 304.99 & 4.17 \\
\hline WW-A & $\mathrm{T} 7$ & $\mathrm{CD}$ & 11.43 & 0.52 & 0.05 & 0.01 & 2.21 & 0.03 & 0.23 & 0.01 & 8.94 & 0.52 & 0.22 & 0.04 & 0.30 & 0.04 & 11.13 & & 23.95 & & -12.82 & & 4.56 & & 1.31 & 0.23 & 6.21 & 0.45 & 1245.32 & 4.32 \\
\hline WW-A & $\mathrm{T} 7$ & $\mathrm{CD}$ & 12.33 & 0.55 & 0.05 & 0.01 & 1.95 & 0.21 & 0.51 & 0.01 & 9.82 & 0.59 & 0.29 & 0.11 & & & 5.15 & & 13.80 & & -8.65 & & 4.44 & & 0.89 & 0.02 & 7.70 & 1.42 & 1775.29 & 6.94 \\
\hline WW-A & $\mathrm{T} 7$ & $\mathrm{C}$ & 14.93 & 0.57 & 0.12 & 0.01 & 2.50 & 0.08 & 0.54 & 0.26 & 11.77 & 0.63 & 0.23 & 0.02 & 0.48 & 0.02 & 8.03 & 4.39 & 22.68 & 1.81 & -14.65 & 2.58 & 4.50 & 0.09 & 0.67 & 0.11 & 7.01 & 0.40 & 299.33 & 3.27 \\
\hline WW-A & $\mathrm{T} 7$ & $\mathrm{CD}$ & 11.88 & 0.38 & 0.05 & 0.00 & 2.08 & 0.11 & 0.37 & 0.10 & 9.38 & 0.40 & 0.26 & 0.06 & 0.30 & 0.04 & 5.15 & & 13.80 & & -8.65 & & 4.44 & & 1.01 & 0.06 & 6.95 & 0.70 & 1245.32 & 4.32 \\
\hline WW-A & $\mathrm{T} 7$ & $1: 10$ & 102.73 & 3.19 & 0.85 & 0.00 & 17.83 & 0.24 & 74.61 & 2.16 & 9.43 & 3.86 & 1.78 & 0.10 & 0.20 & 0.02 & 7.17 & 2.33 & 12.42 & 7.14 & -5.25 & 4.81 & 4.50 & 0.09 & 0.80 & 0.02 & 9.81 & 1.22 & 1024.77 & 72.00 \\
\hline WW-A & $\mathrm{T} 7$ & $1: 5$ & 188.60 & 3.80 & 1.34 & 0.26 & 23.74 & 4.98 & 145.59 & 1.35 & 17.92 & 6.40 & 3.29 & 0.06 & 0.21 & 0.02 & 3.16 & & 10.99 & & -7.83 & & 4.44 & & 0.79 & 0.05 & 6.94 & 0.76 & 1124.37 & 177.22 \\
\hline WW-A & $\mathrm{T} 7$ & IN & 55.32 & 2.42 & 1.72 & 0.02 & 26.59 & 5.87 & 1.45 & 0.67 & 25.55 & 6.38 & 0.30 & 0.07 & 1.32 & 0.07 & & & & & & & & & 0.48 & 0.17 & 8.48 & 0.55 & 708.68 & 39.74 \\
\hline
\end{tabular}




\section{Bray-Curtis distance}
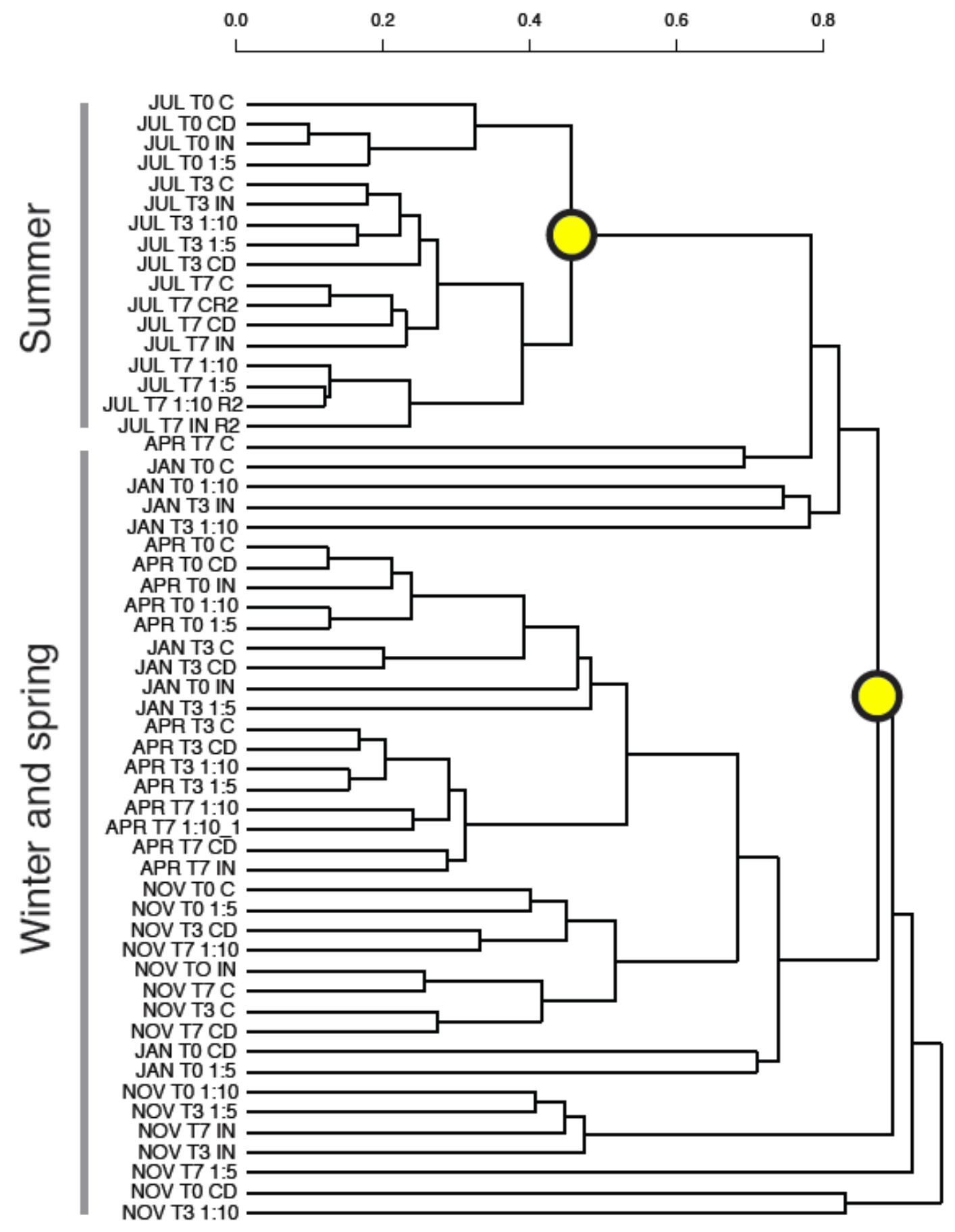

Figure S1. Dendrogram showing a comparison of beta-diversity between samples taken from the experiments performed in January, April, July, November and December. Dissimilarity corresponds to Bray-Curtis distance estimation using 97\% 16S rRNA sequence similarity. Yellow circle denote a distinct visual clustering between summer experiments and winter and spring experiments. 
A

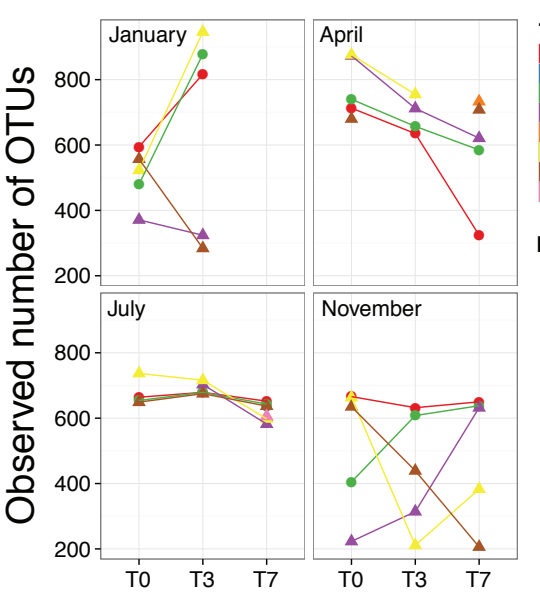

B

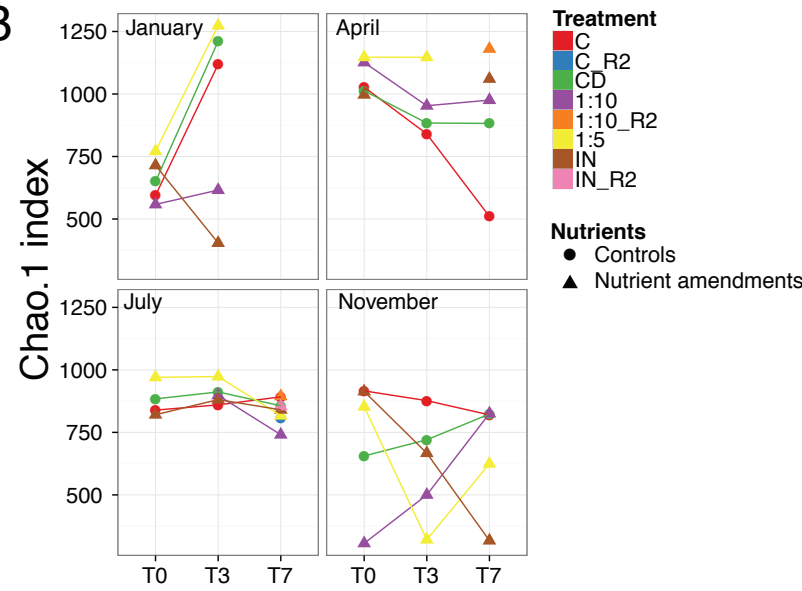

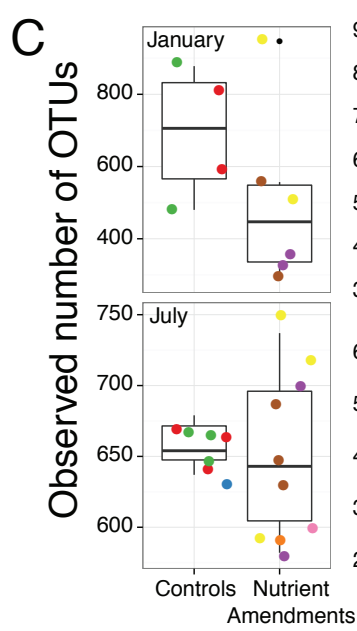

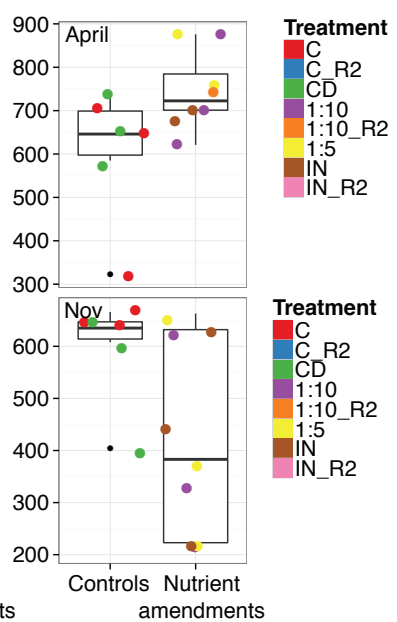

D

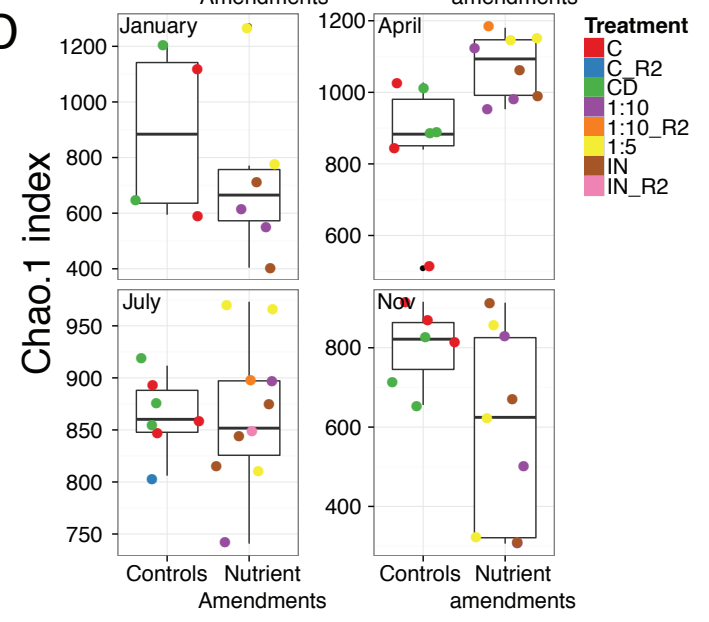

Figure S2. Variation in observed number of OTUs (A) and Chao.1 richness index (B) for the different experiments. 

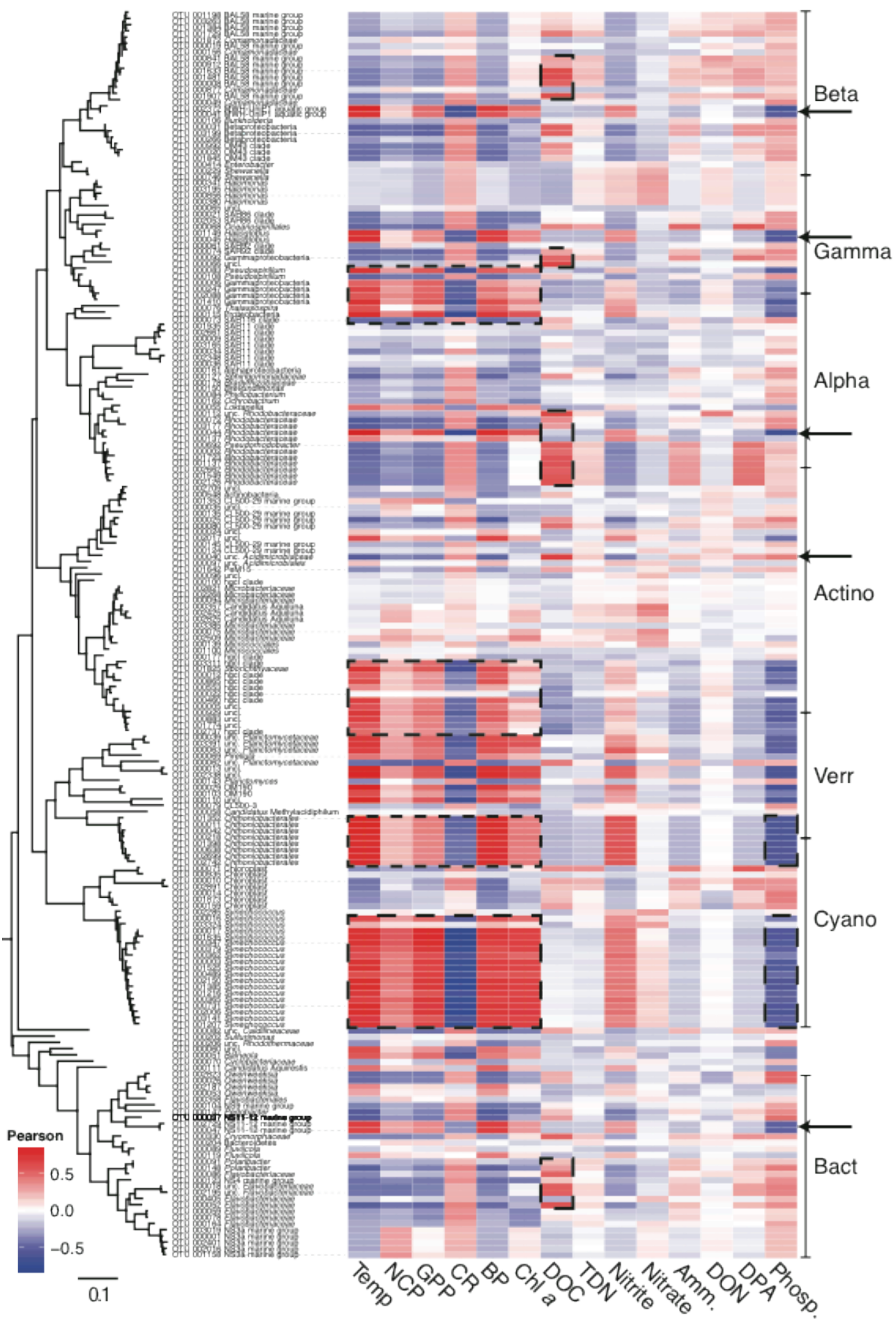

Figure S3. Correlations between shifts in relative abundances of specific individual OTUs and environmental factors and metabolic activity. 

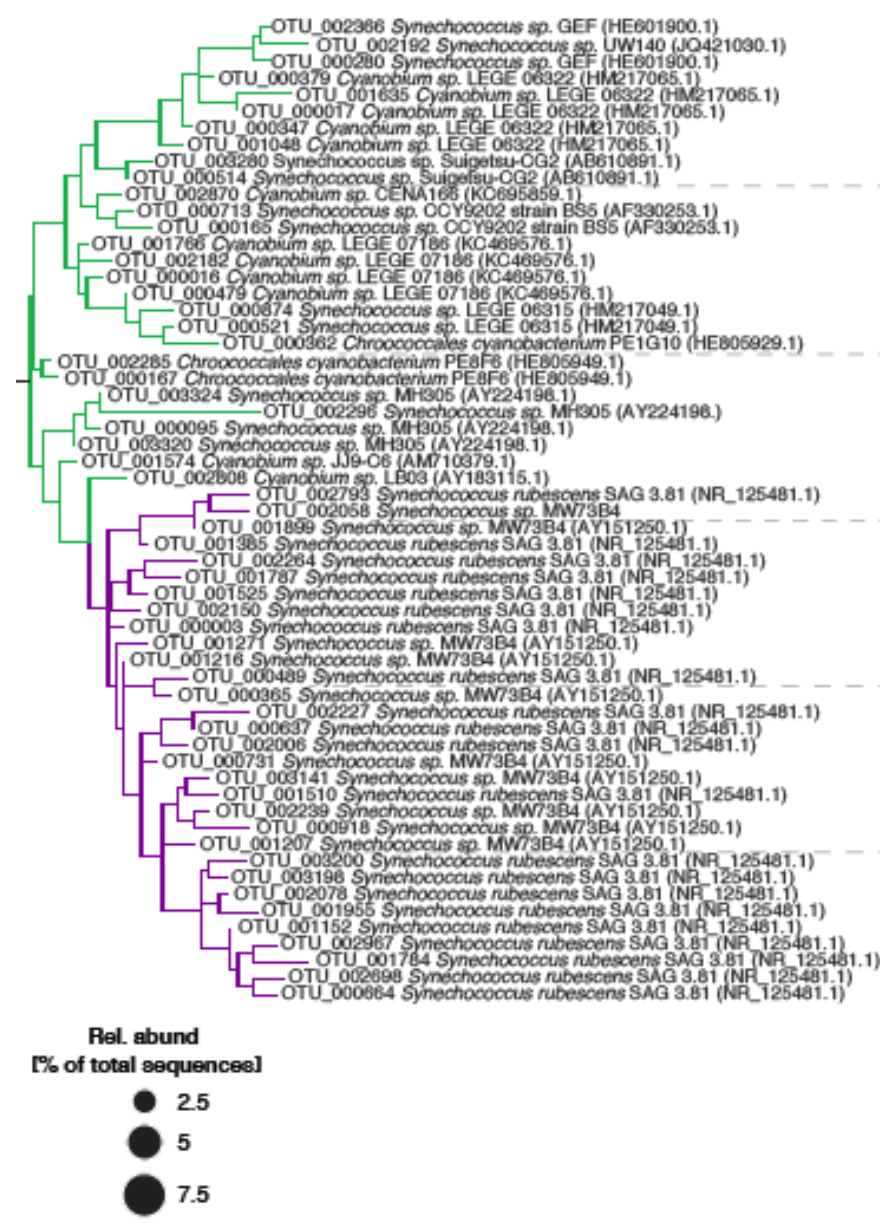

$\stackrel{\longmapsto}{0.01}$

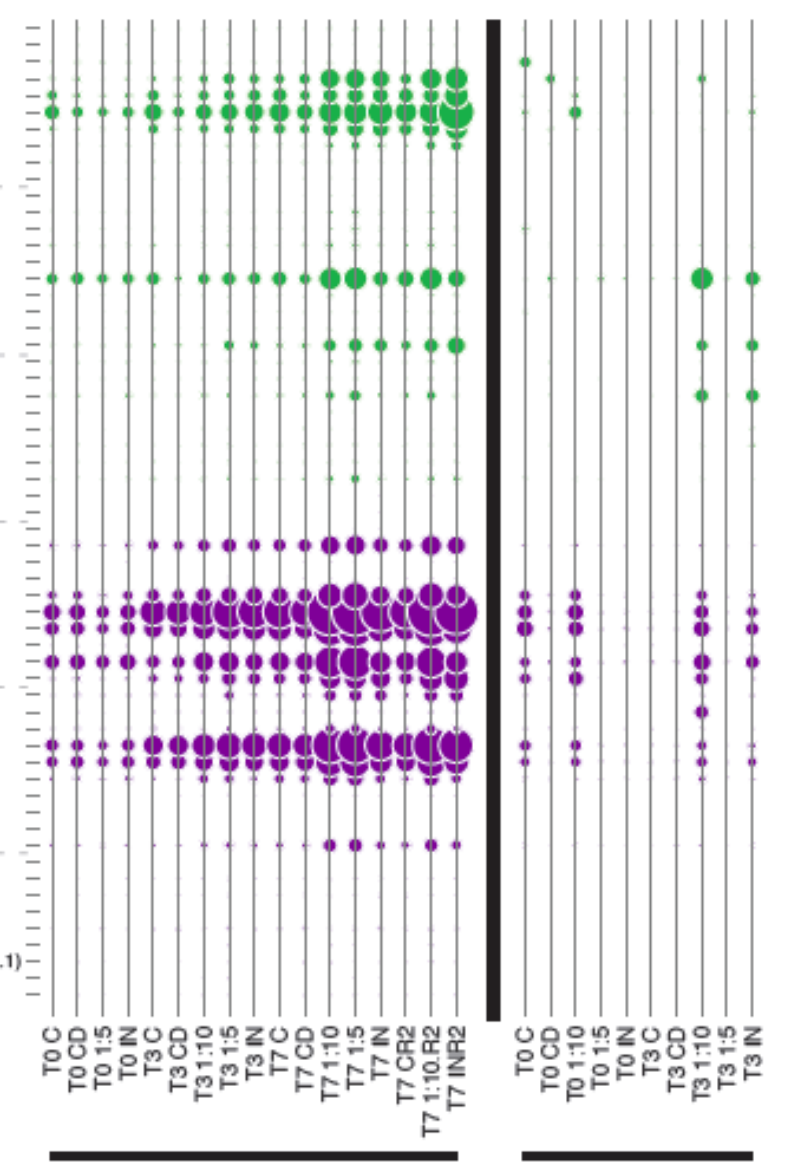

JULY

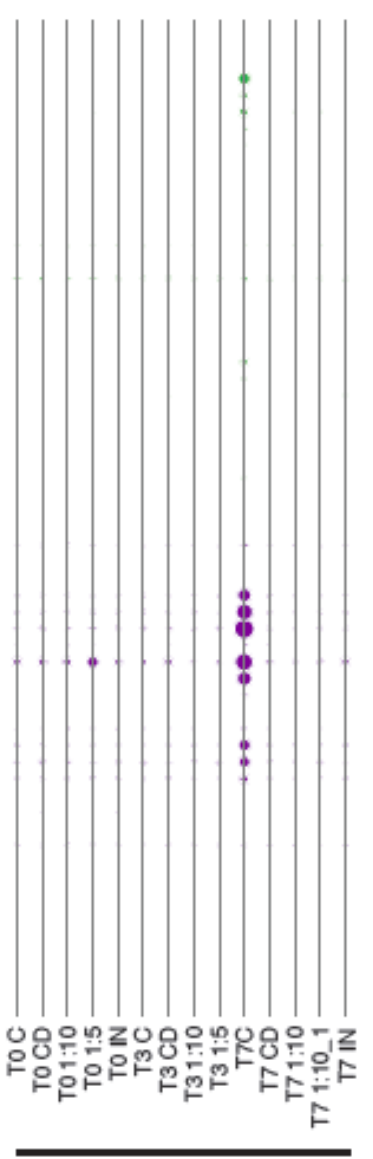

APRIL

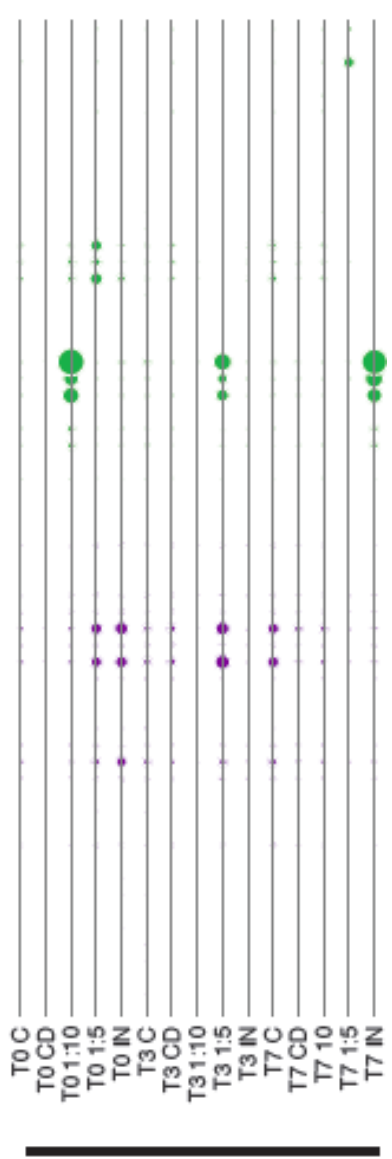

NOVEMBER

Figure S4. Maximum-likelihood tree of 16S rRNA sequences affiliated with Synechococcus obtained from Illumina Miseq-sequencing. Bubble diagram indicate relative abundances (percent of total sequences) in the different treatments and experiments for the 60 most abundant Synechococcus OTUs. 